\title{
WEAK CONVERGENCE OF CD KERNELS AND APPLICATIONS
}

\section{BARRY SIMON}

\begin{abstract}
We prove a general result on equality of the weak limits of the zero counting measure, $d v_{n}$, of orthogonal polynomials (defined by a measure $d \mu$ ) and $(1 / n) K_{n}(x, x) d \mu(x)$. By combining this with the asymptotic upper bounds of Máté and Nevai [16] and Totik [33] on $n \lambda_{n}(x)$, we prove some general results on $\int_{I}(1 / n) K_{n}(x, x) d \mu_{\mathrm{s}} \rightarrow 0$ for the singular part of $d \mu$ and $\int_{I}\left|\rho_{E}(x)-(w(x) / n) K_{n}(x, x)\right| d x \rightarrow 0$, where $\rho_{E}$ is the density of the equilibrium measure and $w(x)$ the density of $d \mu$.
\end{abstract}

\section{Introduction}

We discuss here orthogonal polynomials on the real line (OPRL) and orthogonal polynomials on the unit circle (OPUC) (see [32], [12], [11], [23], [24], [29]). $d \mu$ denotes a measure on $\partial \mathbb{D}=\{z \in \mathbb{C}|| z \mid=1\}$ (positive but not necessarily normalized), $\Phi_{n}(z, d \mu)$ and $\varphi_{n}(z, d \mu)$ denote its monic and normalized OPs and $\left\{\alpha_{n}\right\}_{n=0}^{\infty}$ denotes its Verblunsky coefficients determined by $\left(\Phi_{n}^{*}(z) \equiv z^{n} \overline{\Phi_{n}(1 / \bar{z})}\right)$,

$$
\Phi_{n+1}(z)=z \Phi_{n}(z)-\bar{\alpha}_{n} \Phi_{n}^{*}(z) .
$$

Moreover, with $\rho_{j}=\left(1-\left|\alpha_{j}\right|^{2}\right)^{1 / 2}$,

$$
\left\|\Phi_{n}\right\|_{L^{2}(\partial \mathbb{D}, d \mu)}=\mu(\partial \mathbb{D})^{1 / 2} \prod_{j=0}^{n-1} \rho_{j} .
$$

$d \mu$ also denotes a measure on $\mathbb{R}$ of compact support, and $P_{n}(x, d \mu)$ and $p_{n}(x, d \mu)$ denote its monic and normalized OPs. $\left\{a_{n}, b_{n}\right\}_{n=1}^{\infty}$ are its Jacobi parameters defined by

$$
x p_{n}(x)=a_{n+1} p_{n+1}(x)+b_{n} p_{n}(x)+a_{n} p_{n-1}(x)
$$


and

$$
\left\|P_{n}\right\|_{L^{2}(\mathbb{R}, d \mu)}=\mu(\mathbb{R})^{1 / 2}\left(a_{1} \cdots a_{n}\right) .
$$

The Christoffel [7] and Darboux [8] (CD) kernel is defined by (some authors sum only to $n-1$ )

$$
\begin{aligned}
& K_{n}(z, w)=\sum_{j=0}^{n} \overline{\varphi_{n}(z)} \varphi_{n}(w), \\
& K_{n}(x, y)=\sum_{j=0}^{n} p_{j}(x) p_{j}(y) .
\end{aligned}
$$

The Lebesgue decomposition

$$
\begin{gathered}
d \mu\left(e^{i \theta}\right)=w(\theta) \frac{d \theta}{2 \pi}+d \mu_{\mathrm{s}}\left(e^{i \theta}\right), \\
d \mu(x)=w(x) d x+d \mu_{\mathrm{s}}(x),
\end{gathered}
$$

with $d \mu_{\mathrm{s}}$ Lebesgue singular, enters.

This article concerns both a new method (based on Theorem 1.5) for the study of spectral problems in the theory of OPs and some new results. The method provides new proofs of known results but also new results, of which we emphasize Theorems 1.4 and 7.2 .

To model the issues that concern us here, we recall two consequences of the Szegö condition for OPUC; namely,

$$
\int \log (w(\theta)) \frac{d \theta}{2 \pi}>-\infty .
$$

Here are two central results.

THEOREM 1.1 (Szegö [31])

If the Szegö condition holds, then

$$
\lim _{n \rightarrow \infty} \int\left|\varphi_{n}\left(e^{i \theta}\right)\right|^{2} d \mu_{\mathrm{s}}=0
$$

\section{Remark}

In distinction, if $w=0, \int\left|\varphi_{n}\left(e^{i \theta}\right)\right|^{2} d \mu_{\mathrm{s}} \equiv 1$. 
THEOREM 1.2 (Máté, Nevai, and Totik [18])

If the Szegö condition holds, then for a.e. $\theta$,

$$
w(\theta) \frac{1}{n+1} K_{n}\left(e^{i \theta}, e^{i \theta}\right) \rightarrow 1 .
$$

Máté, Nevai, and Totik state the result in an equivalent form involving the Christoffel function

$$
\lambda_{n}\left(z_{0}\right)=\inf \left\{\int\left|Q_{n}\left(e^{i \theta}\right)\right|^{2} d \mu(\theta) \mid \operatorname{deg} Q_{n} \leq n ; Q_{n}\left(z_{0}\right)=1\right\} .
$$

The minimizer is

$$
\begin{aligned}
Q_{n}\left(e^{i \theta}\right) & =\frac{K_{n}\left(z_{0}, e^{i \theta}\right)}{K_{n}\left(z_{0}, z_{0}\right)}, \\
\lambda_{n}\left(z_{0}\right) & =K_{n}\left(z_{0}, z_{0}\right)^{-1},
\end{aligned}
$$

and since (1.9) implies that $w(\theta)>0$ for a.e. $\theta,(1.11)$ is equivalent to

$$
n \lambda_{n-1}\left(e^{i \theta}\right) \rightarrow w(\theta) .
$$

It is also known under a global condition $w(\theta)>0$ that there are similar results that come from what is called Rakhmanov theory (see [24, Chap. 9] and references therein).

THEOREM 1.3

If $w(\theta)>0$ for a.e. $\theta$, then

(i) (1.10) holds, and

(ii) we have

$$
\left.\lim _{n \rightarrow \infty} \int|w(\theta)| \varphi_{n}\left(e^{i \theta}\right)\right|^{2}-1 \mid \frac{d \theta}{2 \pi}=0 .
$$

\section{Remarks}

(i) is due to Rakhmanov [20], [21].

(ii) is due to Máté, Nevai, and Totik [17].

Theorems 1.1 and 1.2 are known to hold under a local Szegö condition together with regularity in the sense of Stahl and Totik [30] (see also [26] and below).

One of our goals here is to prove the first results of these genres with neither a local Szegö condition nor a global absolute continuity condition. While we focus on the OPRL case, for comparison with Theorems $1-3$, here are our new results for OPUC. 


\section{THEOREM 1.4}

If I is an interval on $\partial \mathbb{D}$ so that

(a) $\quad w(\theta)>0$ a.e. on I; and

(b) $d \mu$ is regular for $\partial \mathbb{D}$; that is,

$$
\left(\rho_{1} \cdots \rho_{n}\right)^{1 / n} \rightarrow 1,
$$

then

(i)

$$
\int_{I} \frac{1}{n+1} K_{n}\left(e^{i \theta}, e^{i \theta}\right) d \mu_{\mathrm{s}}(\theta) \rightarrow 0,
$$

(ii)

$$
\int_{I}\left|1-w(\theta) \frac{1}{n+1} K_{n}\left(e^{i \theta}, e^{i \theta}\right)\right| \frac{d \theta}{2 \pi} \rightarrow 0 .
$$

\section{Remarks}

(1) We do not have pointwise convergence (1.11), but we do have one-half of it; namely, for $e^{i \theta} \in I$,

$$
\liminf w(\theta) \frac{1}{n+1} K_{n}\left(e^{i \theta}, e^{i \theta}\right) \rightarrow 1 .
$$

(2) One associates existence of limits of $\left|\varphi_{n}\right|^{2} d \mu$ with Rakhmanov, which we only expect when $d \mu$ has support on all of $\partial \mathbb{D}$ or a single interval of $\mathbb{R}$. At best, with multiple intervals, one expects almost periodicity of $\left|\varphi_{n}\right|^{2} d \mu$ rather than existence of the limits. For this reason, the Cesàro averages of Theorem 1.4 are quite natural.

There is nothing sacred about $\partial \mathbb{D}$; regularity is defined for any set, and for both OPRL and OPUC, all we need is regularity plus $w(\theta)>0$ on an interval. We also have interesting new bounds on the density of zeros when regularity fails; these generalize a theorem of Totik and Ullman [35].

These new theorems do not involve tweaking the methods used to prove Theorems $1.1-1.3$ but a genuinely new technique (plus one general method of Máté and Nevai [16] used in the proof of Theorem 1.2). Our point here is as much to emphasize this new technique as to prove the results. The new technique is the following.

\section{THEOREM 1.5}

Let $d \mu$ be a measure on $\mathbb{R}$ with bounded support or a measure on $\partial \mathbb{D}$. For $\mathbb{R}$, let $d v_{n}$ be the normalized zero counting measure for the OPRL and for $\partial \mathbb{D}$ for the zeros of the paraorthogonal polynomials (POPUC). Let $n(j)$ be a subsequence 
$n(1)<n(2)<\cdots$. Then for any probability measure, $v_{\infty}$

$$
d v_{n(j)+1} \stackrel{w}{\longrightarrow} d v_{\infty} \Longleftrightarrow \frac{1}{n(j)+1} K_{n(j)}(x, x) d \mu(x) \stackrel{w}{\longrightarrow} d v_{\infty} .
$$

\section{Remarks}

(1) We discuss POPUC and related objects on $\partial \mathbb{D}$ in Section 2.

(2) As we discuss later, $d v_{n(j)}$ and $d v_{n(j)+1}$ have the same limits.

In one sense, this result is more than twenty-five years old. It is a restatement of the invariance of the density of states under change-of-boundary conditions first proved in this context by Avron and Simon [2]. But this invariance is certainly not usually stated in these terms. We also note that for OPUC, we noted this result in [24, Th. 8.2] but did not appreciate its importance.

We note that for OPUC, limits of $(1 /(n+1)) K_{n}\left(e^{i \theta}, e^{i \theta}\right) d \mu(\theta)$ have been studied by Golinskii and Khrushchev [14] without explicitly noting the connection to $\mathrm{CD}$ kernels. Their interesting results are limited to the case of OPUC and mainly to situations where the support is all of $\partial \mathbb{D}$.

By itself, Theorem 1.4 is interesting (e.g., it could be used to streamline the proof of a slightly weaker version of Totik [33, Corollary 2]), but it is really powerful when used with the following collection of results.

THEOREM 1.6 (see Máté and Nevai [16])

For any measure on $\partial \mathbb{D}$,

$$
\limsup n \lambda_{n-1}\left(e^{i \theta}\right) \leq w(\theta)
$$

Máté, Nevai, and Totik [18] noted that this applies to OPRL on $[-1,1]$ using the Szegö mapping, and Totik, in a brilliant article [33], shows how to extend it to any measure of bounded support, $E$, on $\mathbb{R}$ so long as $E$ contains an interval.

THEOREM 1.7 (see Totik [33])

Let $I \subset E \subset \mathbb{R}$, where $I=(a, b)$ is an interval and $E$ is compact. Suppose that $d \mu$ is a measure with support contained in $E$ so that

$$
d \mu(x)=w(x) d x+d \mu_{\mathrm{s}}(x)
$$

Let $d \rho_{E}(x)$ be the potential theoretic equilibrium measure for $E$ (so it is known that $d \rho_{E} \uparrow I=\rho_{E}(x) d x$ for some strictly positive, real-analytic weight $\left.\rho_{E}\right)$. Then for Lebesgue a.e. $x \in I$,

$$
\limsup n \lambda_{n-1}(x) \leq \frac{w(x)}{\rho_{E}(x)} \text {. }
$$




\section{Remarks}

(1) Totik concentrated on the deeper and more subtle fact that if there is a local Szegö condition on $I$ and $\mu$ is regular for $E$, then the limit exists and equals $w(x) / \rho_{E}(x)$ for a.e. $x$. But along the way, he proved (1.24).

(2) We actually prove equality in (1.24) (for $\lim \sup$ ) when $E=\operatorname{supp}(d \mu)$ and $\mu$ is regular.

(3) Later (see Section 8), we prove the analog of Theorem 1.7 for closed subsets of $\partial \mathbb{D}$.

Some of our results assume regularity of $\mu$, so we briefly summarize the main results from that theory, due largely to Stahl and Totik in their book [30]; (see our recent article [26] for an overview).

A measure $\mu$ on $\mathbb{R}$ is called regular if $E=\operatorname{supp}(d \mu)$ is compact and

$$
\lim _{n \rightarrow \infty}\left(a_{1} \cdots a_{n}\right)^{1 / n}=C(E)
$$

where $C(E)$ is the (logarithmic) capacity of $E$. For OPUC, (1.25) is replaced by

$$
\lim _{n \rightarrow \infty}\left(\rho_{1} \cdots \rho_{n}\right)^{1 / n}=C(E) .
$$

Some insight is gained if one knows that in both cases, for any $\mu$ (supported on $E$ compact in $\mathbb{R}$ or $\partial \mathbb{D}$ ), the lim sup is bounded above by $C(E)$. For this article, regularity is important because of the following.

THEOREM 1.8 (see [30, Sec. 2.2], [26, Th. 2.5])

If $d \mu$ is regular, then with $d v_{n}$, the density of zeros of the OPRL or of the POPUC, we have

$$
d v_{n} \stackrel{w}{\longrightarrow} d \rho_{E},
$$

the equilibrium measure for E. Conversely, if (1.27) holds, either $\mu$ is regular or $\mu$ is supported on a set of capacity zero.

We now mention one criterion for regularity that goes back to Erdös and Turán [10] for $[-1,1]$ and Widom [37] for general $E$.

THEOREM 1.9

If $E=\operatorname{supp}(d \mu)$ and

$$
d \mu(x)=f(x) d \rho_{E}(x)+d \mu_{\mathrm{s}}(x)
$$

where $\mu_{\mathrm{s}}$ is $\rho_{E}$-singular and $f(x)>0$ for $\rho_{E}$-a.e. $x$, then $\mu$ is regular. 
There is a proof of Van Assche [36] of the general case presented in [26] which is not difficult. But in the case where $E^{\text {int }}$ (interior in the sense of $\mathbb{R}$ ) differs from $E$ by a set of capacity zero (e.g., $E=[-1,1]$ ), we find a proof that uses only our strategy in Theorems 1.5 and 1.7. In particular, we have a proof of the Erdös-Turán result in [10] which uses neither potential theory nor polynomial inequalities.

We can now describe the content of this article. In Section 2, we prove the main weak convergence result, Theorem 1.5. In Section 3, we prove the analog of the Erdös-Turán result for $\partial \mathbb{D}$ and illustrate how these ideas are connected to the regularity criterion of Stahl and Totik [30]. Section 4 proves Theorem 1.4 for $\mu$-regular on $\partial \mathbb{D}$. Sections 5 and 6 then parallel Sections 3 and 4 but for general compact sets $E \subset \mathbb{R}$. Section 7, motivated by work of Totik and Ullman in [35], provides a comparison result about densities of zeros. Section 9 does the analog of Sections 5 and 6 for general $E \subset \partial \mathbb{D}$. To do this, we need Totik's result (1.24) in that situation. This does not seem to be in the literature, so Section 8 fills that need.

\section{Weak convergence}

Our main goal here is to prove a generalization of Theorem 1.5 (and so, also that theorem). We let $\mu$ be a measure of compact support in $\mathbb{C}$, and we let

$$
N(\mu)=\sup \{|z| \mid z \in \operatorname{supp}(d \mu)\} .
$$

We let $M_{z}$ be multiplication by $z$ on $L^{2}(\mathbb{C}, d \mu)$, so

$$
\left\|M_{z}\right\|=N(\mu)
$$

and we let $Q_{n}$ be the $(n+1)$-dimensional orthogonal projection onto polynomials of degree $n$ or less. All estimates here depend on the following.

PROPOSITION 2.1

Fix $\ell=1,2, \ldots$ Then

$$
Q_{n} M_{z}^{\ell} Q_{n}-\left(Q_{n} M_{z} Q_{n}\right)^{\ell}
$$

is an operator of rank at most $\ell$ and norm at most $2 N(\mu)^{\ell}$.

\section{Proof}

Let $X$ be the operator in (2.3). Clearly, $X=0$ on $\operatorname{ran}\left(1-Q_{n}\right)=\operatorname{ker}\left(Q_{n}\right)$. Since $M_{z}$ maps $\operatorname{ran}\left(Q_{j}\right)$ to $\operatorname{ran}\left(Q_{j+1}\right), X=0$ on $\operatorname{ran}\left(Q_{n-\ell}\right)$. This shows that $X$ has rank at most $\ell$. The norm estimate is immediate from (2.2).

Here is the link to $K_{n}$ and to $d v_{n+1}$. Let $X_{j}(z, d \mu)$ be the monic OPs for $\mu$, and let $x_{n}=X_{n} /\left\|X_{n}\right\|$. 
PROPOSITION 2.2

(i) We have, for all $w \in \mathbb{C}$,

$$
\operatorname{det}_{Q_{n}}\left(w-Q_{n} M_{z} Q_{n}\right)=X_{n+1}(w, d \mu)
$$

In particular, if $d v_{n+1}$ is the zero counting measure for $X_{n+1}$, then

$$
\frac{1}{n+1} \operatorname{Tr}\left(\left(Q_{n} M_{z} Q_{n}\right)^{\ell}\right)=\int z^{\ell} d v_{n+1}(z) .
$$

(ii) Let

$$
K_{n}(z, w)=\sum_{j=0}^{n} \overline{x_{j}(z)} x_{j}(w)
$$

Then

$$
\operatorname{Tr}\left(Q_{n} M_{z}^{\ell} Q_{n}\right)=\int z^{\ell} K_{n}(z, z) d \mu(z) .
$$

Remark. The proof of (i) is due to Davies and Simon [9].

Proof of Proposition 2.2

(i) Let $z_{0}$ be a zero of $X_{n+1}$ of order $\ell$. Let $\varphi(z)=X_{n+1}(z) /\left(z-z_{0}\right)^{\ell}$. Since $Q_{n}\left[X_{n+1}\right]=$ 0 , we see, with $M_{z}^{(n)}=Q_{n} M_{z} Q_{n}$,

$$
\left(M_{z}^{(n)}-z_{0}\right)^{\ell} \varphi=0, \quad\left(M_{z}^{(n)}-z_{0}\right)^{\ell-1} \varphi \neq 0
$$

showing that $z_{0}$ is a zero of $\operatorname{det}_{Q_{n}}\left(w-M_{z}^{(n)}\right)$ of order at least $\ell$. In this way, we see that $X_{n+1}(w)$ and the det have the same zeros. Since both are monic, we obtain (2.4).

In particular, this shows

$$
\operatorname{Tr}\left(\left(M_{z}^{(n)}\right)^{\ell}\right)=\sum_{\substack{\text { zeros } z_{j} \text { of } \\ \text { multiplicity } m_{j}}} m_{j} z_{j}^{\ell}=(n+1) \int z^{\ell} d v_{n+1}(z),
$$

proving (2.5).

(ii) In $L^{2}(\mathbb{C}, d \mu),\left\{x_{j}\right\}_{j=0}^{n} \operatorname{span} \operatorname{ran}\left(Q_{n}\right)$ and are an orthonormal basis, so

$$
\begin{aligned}
\operatorname{Tr}\left(Q_{n} M_{z}^{\ell} Q_{n}\right) & =\sum_{j=0}^{n}\left\langle x_{j}, z^{\ell} x_{j}\right\rangle \\
& =\int \sum_{j=0}^{n} z^{\ell}\left|x_{j}(z)\right|^{2} d \mu(z),
\end{aligned}
$$

proving (2.7). 
PROPOSITION 2.3

Let $d \eta_{n}$ be the probability measure

$$
d \eta_{n}(z)=\frac{1}{n+1} K_{n}(z, z) d \mu(z)
$$

Then for $\ell=0,1,2, \ldots$,

$$
\left|\int z^{\ell} d \eta_{n}(z)-\int z^{\ell} d v_{n+1}(z)\right| \leq \frac{2 \ell N(\mu)^{\ell}}{n+1} .
$$

Suppose that there is a compact set, $K \subset \mathbb{C}$, containing the supports of all $d v_{n}$ and the support of $d \mu$ such that $\left\{z^{\ell}\right\}_{\ell=0}^{\infty} \cup\left\{\bar{z}^{\ell}\right\}_{\ell=0}^{\infty}$ are $\|\cdot\|_{\infty}$-total in the continuous function on $K$. Then for any subsequence $n(j), d \eta_{n(j)} \rightarrow d \nu_{\infty}$ if and only if $d v_{n(j)+1} \rightarrow d v_{\infty}$.

Proof

(2.10) is immediate from (2.5) and (2.7) if we note that, by Proposition 2.1,

$$
\left|\operatorname{Tr}\left(Q_{n} M_{z}^{\ell} Q_{n}\right)-\operatorname{Tr}\left(\left(Q_{n} M_{z} Q_{n}\right)^{\ell}\right)\right| \leq 2 N(\mu)^{\ell} \ell .
$$

In turn, (2.10) implies that we have the weak convergence result.

While we were careful to use $n(j)$ and $n(j)+1$, we note that since $\left|x_{j}(z)\right|^{2} d \mu$ is a probability measure, we have

$$
\left\|\eta_{n}-\eta_{n+1}\right\| \leq \frac{1}{n+1}+n\left(\frac{1}{n}-\frac{1}{n+1}\right) \leq \frac{2}{n+1},
$$

so we could just as well have discussed weak limits of $\eta_{n(j)+1}$ and $v_{n(j)+1}$. The following is Theorem 1.5 for OPRL.

\section{THEOREM 2.4}

For OPRL, $d \eta_{n(j)}$ converges weakly to $d v_{\infty}$ if and only if $d v_{n(j)+1}$ converges weakly to $d v_{\infty}$.

\section{Proof}

$\left\{x_{j}\right\}_{j=0}^{\infty}$ are total in $C([\alpha, \beta])$ for any real interval $[\alpha, \beta]$, so Proposition 2.3 is applicable.

For OPUC, we need a few preliminaries. Let $\mathscr{M}_{+, 1}(X)$ be the probability measures on a compact set $X$. Define $\mathscr{P}: C(\partial \mathbb{D}) \rightarrow C(\mathbb{D})$ (with $\mathbb{D}=\{z|| z \mid \leq 1\}$ ) by

$$
(\mathscr{P} f)\left(r e^{i \theta}\right)=\int \frac{1-r^{2}}{1+r^{2}-2 r \cos (\theta-\varphi)} f\left(e^{i \theta}\right) \frac{d \varphi}{2 \pi}
$$


for $r<1$ and $(\mathscr{P} f)\left(e^{i \theta}\right)=f\left(e^{i \theta}\right)$. We also define $\mathscr{P}^{*}: \mathscr{M}_{+, 1}(\overline{\mathbb{D}}) \rightarrow \mathscr{M}_{+, 1}(\partial \mathbb{D})$, the balayage, by duality. Then the following is well known and elementary.

PROPOSITION 2.5

$\mathscr{P}^{*}(d \nu)$ is the unique measure, $\eta$, on $\partial \mathbb{D}$ with

$$
\int e^{i \ell \theta} d \eta(\theta)=\int z^{\ell} d v(z)
$$

for $\ell=0,1,2, \ldots$

Widom [37] proved that if $\mu$ is supported on a strict subset of $\partial \mathbb{D}$, then the zero counting measure, $d v_{n}$, has weak limits supported on $\partial \mathbb{D}$,

$$
\mathscr{P}^{*}\left(d v_{n}\right)-d v_{n} \stackrel{w}{\longrightarrow} 0 .
$$

Finally, we note the following about POPUC defined for $\beta \in \partial \mathbb{D}$ by

$$
P_{n+1}(z, \beta)=z \Phi_{n}(z)-\bar{\beta} \Phi_{n}^{*}(z)
$$

defined in [15] and studied further in [4]- [6], [13], [27], [38].

PROPOSITION 2.6

Let $\beta_{n}$ be an arbitrary sequence $\partial \mathbb{D}$, and let $d \nu_{n+1}^{\left(\beta_{n}\right)}$ be the zero counting measure for $P_{n+1}\left(z, \beta_{n}\right)$ (known to live on $\partial \mathbb{D}$; see, e.g., [27]). Then for any $\ell \geq 0$,

$$
\left|\int z^{\ell} d \nu_{n+1}-\int z^{\ell} d \nu_{n+1}^{\left(\beta_{n}\right)}\right| \rightarrow 0 .
$$

\section{Proof}

Let $\mathscr{C}_{n+1, F}$ be the truncated CMV matrix of size $n+1$ whose eigenvalues are the zeros of $\Phi_{n+1}(z)$ (see [23, Chap. 4]), and let $\mathscr{C}_{n+1, F}^{\left(\beta_{n}\right)}$ be the unitary dilation whose eigenvalues are the zeros of $P_{n+1}\left(z, \beta_{n}\right)$ (see [6], [27], [25]). Then $\mathscr{C}_{n+1, F}-\mathscr{C}_{n+1, F}^{\left(\beta_{n}\right)}$ is rank 1 with norm bounded by 2 , so $\mathscr{C}_{n+1, F}^{\ell}-\left[\mathscr{C}_{n+1, F}^{(\beta)}\right]^{\ell}$ is rank at most $\ell$ with norm 2. Thus

$$
\left|\operatorname{Tr}\left(\mathscr{C}_{n+1, F}^{\ell}-\left(\mathscr{C}_{n+1, F}^{(\beta)}\right)^{\ell}\right)\right| \leq 2 \ell
$$

and

$$
\mid \text { LHS of (2.17) } \mid \leq \frac{2 \ell}{n+1},
$$

proving (2.17). 


\section{THEOREM 2.7}

For OPUC, $d \eta_{n(j)}$ converges weakly to $d v_{\infty}$ if and only if each of the following converges weakly to $d \nu_{\infty}$ :

(i) $\quad d v_{n+1}^{\left(\beta_{n}\right)}$ for any $\beta_{n}$;

(ii) $\mathscr{P}\left(d v_{n+1}\right)$, the balayage of $d v_{n+1}$;

(iii) $\quad d v_{n+1}$ if $\operatorname{supp}(d \mu) \neq \partial \mathbb{D}$.

\section{Proof}

This is immediate from Propositions 2.3 and 2.6.

When I mentioned Theorem 2.4 to Vilmos Totik, he found an alternate proof, using tools more familiar to OP workers, which provides some insight. With his permission, I include this proof. Let me discuss the result for convergence of sequences rather than subsequences and then give some remarks to handle subsequences.

The key to Totik's proof is Gaussian quadratures, which say that if $\left\{x_{j}^{(n)}\right\}_{j=1}^{n}$ are the zeros of $p_{n}(x, d \mu)$ and $\lambda_{n-1}(x)=K_{n-1}(x, x)^{-1}$, then for any polynomial $R_{m}$ of degree $m \leq 2 n-1$,

$$
\int R_{m}(x) d \mu(x)=\sum_{j=1}^{n} \lambda_{n-1}\left(x_{j}^{(n)}\right) R_{m}\left(x_{j}^{(n)}\right) .
$$

We also need the Christoffel variation principle,

$$
n \leq q \Rightarrow \lambda_{q}(x) \leq \lambda_{n}(x)
$$

which is immediate from (1.12).

Suppose we know that $d v_{n} \rightarrow d \nu_{\infty}$. Fix a polynomial $Q_{m}$ of degree $m$ with $Q_{m} \geq 0$ on $\operatorname{cvh}(\operatorname{supp}(d \mu))$, where cvh $=$ convex hull. Let

$$
R_{m+2 n}(x)=\frac{1}{n+1} Q_{m}(x) K_{n}(x, x)
$$

which has degree $m+2 n$. Thus, if $N=n+m$, we have, by (2.19),

$$
\begin{aligned}
\int Q_{m}(x) & {\left[\frac{1}{n+1} K_{n}(x, x)\right] d \mu } \\
& =\sum_{j=1}^{N} \lambda_{N-1}\left(x_{j}^{(N-1)}\right) Q_{m}\left(x_{j}^{(N-1)}\right)\left(\frac{1}{n+1}\right) \lambda_{n}\left(x_{j}^{(N-1)}\right)^{-1} \\
& \leq\left(\frac{N+1}{n+1}\right) \frac{1}{N+1} \sum_{j=1}^{N} Q_{m}\left(x_{j}^{(N-1)}\right)
\end{aligned}
$$


by $Q_{m} \geq 0$ and (2.21) (so $\lambda_{N-1} / \lambda_{n} \leq 1$ ). As $n \rightarrow \infty, N / n \rightarrow 1$. So, by hypothesis,

$$
\text { RHS of (2.22) } \rightarrow \int Q_{m}(y) d v_{\infty}(y) .
$$

We conclude, by (2.21), that

$$
\lim \sup \int Q_{m}(x) d \eta_{n}(x) \leq \int Q_{m}(y) d v_{\infty}(y) .
$$

If $0 \leq Q_{m} \leq 1$ on $\operatorname{cvh}(\operatorname{supp}(d \mu))$, we can apply this also to $1-Q_{m}$ and so conclude for such $Q_{m}$ that

$$
\lim \int Q_{m} d \eta_{n}(x)=\int Q_{m} d v_{\infty},
$$

which implies that w-lim $d \eta_{n}=d v_{\infty}$.

The same inequality (2.22) can be used to show that if $d \eta_{n} \stackrel{w}{\longrightarrow} d \eta_{\infty}$, then

$$
\liminf \int Q_{m}(y) d v_{m}(y) \geq \int Q_{m}(y) d \eta_{\infty}(y),
$$

and thus, by the same $1-Q$ trick, we get $d v_{n} \rightarrow d \eta_{\infty}$.

To handle subsequences, we need only note that by (2.12), if $d \eta_{n(j)} \rightarrow d \eta_{\infty}$, then $d \eta_{n(j)+\ell} \rightarrow d \eta_{\infty}$ for $\ell=0, \pm 1, \pm 2, \ldots$ Similarly, by zero interlacing, if $d v_{n(j)} \rightarrow d v_{\infty}$, then $d v_{n(j)+\ell} \rightarrow d v_{\infty}$ for fixed $\ell$.

By using the operator theoretic proof of Gaussian quadrature (see, e.g., [23, Sec. 1.2]), one sees that this proof is closely related to our proof above.

\section{Regularity for $\partial \mathbb{D}$ : The Erdös-Turán theorem}

Our goal in this section is to prove the following.

\section{THEOREM 3.1}

Let $d \mu$ on $\partial \mathbb{D}$ have the form (1.7) with $w(\theta)>0$ for a.e. $\theta$. Then $\mu$ is regular; that is, (1.26) holds with $E=\partial \mathbb{D}$ ( $\operatorname{so} C(E)=1)$.

\section{Remarks}

(1) This is an analog of a theorem for [-1, 1] proven by Erdös and Turán [10]. Our proof here seems to be new.

(2) This is, of course, weaker than Rakhmanov's theorem (see [24, Chap. 9] and references therein), but as we see, this extends easily to some other situations. 
The proof combines the Máté-Nevai theorem (Theorem 1.6) and Proposition 2.5. It is worth noting where the Máté-Nevai theorem comes from. By (1.12), if

$$
Q_{n}\left(e^{i \theta}\right)=\frac{1}{n+1} \sum_{j=0}^{n} e^{i j(\theta-\varphi)}
$$

then

$$
\lambda_{n}\left(e^{i \varphi}\right) \leq \int\left|Q_{n}\left(e^{i \theta}\right)\right|^{2} d \mu(\theta)
$$

Recognizing $(n+1)\left|Q_{n}\right|^{2}$ as the Fejér kernel, (1.22) is a standard maximal function a.e. convergence result.

PROPOSITION 3.2

For any measure on $\partial \mathbb{D}$ for a.e. $\theta$,

$$
\liminf _{n \rightarrow \infty} \frac{1}{n+1} K_{n}\left(e^{i \theta}, e^{i \theta}\right) \geq w(\theta)^{-1} .
$$

On the set where $w(\theta)>0$,

$$
\liminf _{n \rightarrow \infty} \frac{1}{n+1} w(\theta) K_{n}\left(e^{i \theta}, e^{i \theta}\right) \geq 1 .
$$

Remark. If $w(\theta)=0,(3.3)$ is interpreted as saying that the limit is infinite. In that case, of course, (3.4) does not hold.

\section{Proof}

We have that (3.3) is immediate from (1.14) and (1.22). If $w \neq 0, w w^{-1}=1,(3.3)$ implies (3.4).

\section{Proof of Theorem 3.1}

The hypothesis $w>0$ for a.e. $\theta$ implies that $d \mu$ is not supported on a set of capacity zero. Thus, by Theorem 1.8, regularity holds if we prove that the density of zeros of POPUC converges to $\frac{d \theta}{2 \pi}$. By Proposition 2.5, this follows if we prove that $(1 /(n+1)) K_{n+1} d \mu \rightarrow \frac{d \theta}{2 \pi}$.

Suppose that $n(j) \rightarrow \infty$ is a subsequence with $(1 /(n+1)) K_{n+1}\left(e^{i \theta}, e^{i \theta}\right) w(\theta)$ $\frac{d \theta}{2 \pi} \rightarrow d \nu_{1}$ and $(1 /(n+1)) K_{n+1}\left(e^{i \theta}, e^{i \theta}\right) d \mu_{\mathrm{s}} \rightarrow d \nu_{2}$. Then since $(1 /(n+1)) K_{n} d \mu$ is normalized,

$$
\int\left[d v_{1}+d v_{2}\right]=1
$$


On the other hand, by Fatou's lemma and (3.4), for any continuous $f \geq 0$ and the hypothesis $w(\theta)>0$ a.e. $\theta$,

$$
\begin{aligned}
\int f d v_{1} & =\lim \int f\left[\frac{1}{n+1} w(\theta) K_{n}\left(e^{i \theta}, e^{i \theta}\right)\right] \frac{d \theta}{2 \pi} \\
& \geq \int \liminf \left[f \frac{1}{n+1} w(\theta) K_{n}\left(e^{i \theta}, e^{i \theta}\right)\right] \frac{d \theta}{2 \pi} \\
& \geq \int f(\theta) \frac{d \theta}{2 \pi} .
\end{aligned}
$$

Thus

$$
d \nu_{1} \geq \frac{d \theta}{2 \pi}
$$

By (3.5), this can happen only if

$$
d \nu_{1}=\frac{d \theta}{2 \pi}, \quad d \nu_{2}=0 .
$$

As a result of the compactness of the space of measures, one has that $(1 /(n+1))$ $K_{n+1} d \mu \rightarrow \frac{d \theta}{2 \pi}$.

Along the way, we also proved that $d v_{2}=0$; that is, we proved the following.

\section{THEOREM 3.3}

Under the hypotheses of Theorem 3.1,

$$
\frac{1}{n+1} \int \sum_{j=0}^{n}\left|\varphi_{j}\left(e^{i \theta}\right)\right|^{2} d \mu_{\mathrm{s}}(\theta) \rightarrow 0 .
$$

It would be interesting to see if these methods provide an alternate proof of the theorem of Stahl and Totik [30, Th. 4.2.7] that if for all $\eta>0$,

$$
\lim _{n \rightarrow \infty}\left|\left\{\theta|| \mu\left(\mid\left\{\psi|| e^{i \theta}-e^{i \psi}\left|\leq \frac{1}{n}\right|\right\}\right) \leq e^{-n \eta}\right\}\right|=0
$$

then $\mu$ is regular. The point is that using powers of the Fejér kernel, one can get trial functions localized in an interval of size $O(1 / n)$, and off a bigger interval of size $O(1 / n)$, it is exponentially small. (3.9) should say that the dominant contribution comes from an $O(1 / n)$-interval. On the other hand, the translates of these trial functions are spread over $O(1 / n)$-intervals, so one gets lower bounds on $(1 / n) K_{n}\left(e^{i \theta}, e^{i \theta}\right)$ 
of order $\mu\left(e^{i \theta}-c / n, e^{i \theta}+c / n\right)^{-1}$ which are then integrated against $d \mu$, canceling this inverse and hopefully leading to (3.7), and so regularity.

\section{Localization on $\partial \mathbb{D}$}

In this section, we prove Theorem 1.4. Instead of using the Máté-Nevai bound to prove regularity, we combine it with regularity to get information.

Proof of Theorem 1.4

As in the proof of Theorem 3.1, we let $d v_{1}, d \nu_{2}$ be weak limits of $(1 / n) K\left(e^{i \theta}, e^{i \theta}\right) w(\theta) \frac{d \theta}{2 \pi}$ and $(1 / n) K_{n}\left(e^{i \theta}, e^{i \theta}\right) d \mu_{\mathrm{s}}(\theta)$. On $I$, the same arguments as above imply that

$$
d \nu_{1} \uparrow I \geq \frac{d \theta}{2 \pi} \uparrow I
$$

By regularity, globally,

$$
d \nu_{1}+d \nu_{2}=\frac{d \theta}{2 \pi}
$$

Thus, on $I$,

$$
v_{1}\left\lceil I=\frac{d \theta}{2 \pi}, \quad v_{2}\lceil I=0 .\right.
$$

The second implies (1.18). The first implies that

$$
\int_{a}^{b} w(\theta) \frac{1}{n+1} K_{n}\left(e^{i \theta}, e^{i \theta}\right) \frac{d \theta}{2 \pi} \rightarrow(b-a) .
$$

Since (3.4) and Fatou imply that

$$
\int_{a}^{b}\left[w(\theta) \frac{1}{n+1} K_{n}\left(e^{i \theta}, e^{i \theta}\right)-1\right]_{-} \frac{d \theta}{2 \pi} \rightarrow 0
$$

we obtain (1.19).

Notice that (4.4) and (3.4) imply a pointwise a.e. result (which we stated as (1.20)),

$$
\liminf _{n \rightarrow \infty} \frac{1}{n+1} w(\theta) K_{n}\left(e^{i \theta}, e^{i \theta}\right)=1 .
$$

We do not know how to get a pointwise result on lim sup under only the condition $w(\theta)>0$ (but without a local Szegö condition). 


\section{Regularity for $E \subset \mathbb{R}$ : Widom's theorem}

In this section and Section 6, our goal is to extend the results of Sections 3 and 4 to situations where $\partial \mathbb{D}$ is replaced by fairly general closed sets in $\mathbb{R}$. The keys are Theorem 2.4 and Totik's Theorem 1.7. We begin with a few remarks on where Theorem 1.7 comes from (see also Section 8).

Since the hypothesis is that $\operatorname{supp}(d \mu) \subset E$, not equal to $E$, it suffices to find $E_{n} \supset E$ so that $\rho_{E_{n}}(x) \rightarrow \rho_{E}(x)$ on $I$ and for which (1.21) can be proved. By using $\tilde{E}_{n}=\{x \mid \operatorname{dist}(x, E) \leq 1 / n\}$, one first gets approximation by a finite union of intervals, and then, by a theorem proven by Bogatyrëv [3], Peherstorfer [19], and Totik [34], one finds $\tilde{E}_{n} \subset E_{n}$, where the $E_{n}$ 's are finite unions of intervals with rational harmonic measure. For rational harmonic measures, one can use as trial polynomials $K_{m}\left(x, x_{0}\right) / K_{m}\left(x_{0}, x_{0}\right)$, where $K_{m}$ is the CD kernel of a measure in the periodic isospectral torus and Floquet theory. (This is the method from Simon [28]; Totik [33] instead uses polynomial mapping.)

\section{THEOREM 5.1}

Let $E \subset \mathbb{R}$ be a compact set with $\partial E \equiv E \backslash E^{\mathrm{int}}\left(E^{\mathrm{int}}\right.$ means interior in $\left.\mathbb{R}\right)$ having capacity zero (e.g., a finite union of closed intervals). Let $d \mu$ be a measure with $\sigma_{\mathrm{ess}}(d \mu)=E$ and

$$
d \mu=f(x) d \rho_{E}+d \mu_{\mathrm{s}}
$$

where $d \mu_{\mathrm{s}}$ is $d \rho_{E}$-singular. Suppose that $f(x)>0$ for $d \rho_{E}$-a.e. $x$. Then $d \mu$ is regular.

\section{Remarks}

(1) In this case, $d \rho_{E}$ is equivalent to $\chi_{E} d x$.

(2) For any compact $E$, this is a result of Widom [37] (see also Van Assche [36], Stahl and Totik [30], and Simon [26]).

\section{Proof of Theorem 5.1}

Essentially identical to Theorem 3.1. By Theorem 1.8 and the fact that $d \mu$ is clearly not supported on sets of capacity zero, it suffices to prove that $d v_{n} \rightarrow d \rho_{E}$. Pick $n(j) \rightarrow$ $\infty$, so $(1 /(n(j)+1)) K_{n(j)}(x, x) f(x) d \rho_{E}$ and $(1 /(n(j)+1)) K_{n(j)}(x, x) d \mu_{\text {s }}$ separately have limits $d v_{1}$ and $d v_{2}$. It says in (1.21) that (given that $f(x)>0$ for a.e. $x$ )

$$
\liminf \frac{1}{n(j)+1} K_{n(j)}(x, x) f(x) \geq 1
$$

By Fatou's lemma on $E^{\text {int}}$,

$$
d \nu_{1} \geq d \rho_{E} .
$$


Also, since $\int\left(d \nu_{1}+d \nu_{2}\right)=1$ and $\int_{E^{\text {int }}} d \rho_{E}=1$ (since $\left.C\left(E \backslash E^{\text {int }}\right)=0\right)$, we conclude that $d v_{1}=d \rho_{E}, d \nu_{2}=0$. By compactness of probability measures, $(1 /(n(j)+1)) K_{n(j)}(x, x) d \mu \stackrel{w}{\longrightarrow} d \rho_{E}$, implying regularity.

\section{Localization on $\mathbb{R}$}

Here is an analog of Theorem 1.4 for any $E \subset \mathbb{R}$.

THEOREM 6.1

Let $I=[a, b] \subset E \subset \mathbb{R}$ with $a<b$ and $E$ compact. Let $d \mu$ be a measure on $\mathbb{R}$ so that $\sigma_{\mathrm{ess}}(d \mu)=E$ and $\mu$ is regular for $E$. Suppose that

$$
d \mu=w(x) d x+d \mu_{\mathrm{s}}
$$

with $d \mu_{\mathrm{s}}$ Lebesgue singular. Suppose that $w(x)>0$ for a.e. $x \in I$. Then

(i) $\quad(1 /(n+1)) K_{n}(x, x) d \mu_{\mathrm{s}} \stackrel{w}{\longrightarrow} 0$;

(ii) $\quad \int_{I}\left|\rho_{E}(x)-(1 /(n+1)) w(x) K_{n}(x, x)\right| d x \rightarrow 0$.

Proof

By $w(x)>0$ a.e. on $I$ and (1.17),

$$
\liminf w(x) \frac{1}{n+1} K_{n}(x, x) \geq \rho_{E}(x)
$$

for a.e. $x \in I$. From this, one can follow exactly the proofs in Section 4 .

\section{Comparisons of density of zeros}

In [35], Totik and Ullman proved the following. (We take $[-a, a]$ rather than $[a, b]$ only for notational simplicity.)

THEOREM 7.1 (see [35])

Let $d \mu$ be a measure supported on a subset of $[-1,1]$, where

$$
d \mu=w(x) d x+d \mu_{\mathrm{s}}
$$

with

$$
w(x)>0 \text { for a.e. } x \in[-a, a]
$$

for some $a \in(0,1)$. Let $d \nu_{\infty}$ be any limit point of the zero counting measures for $d \mu$. Then on $(-a, a)$, we have

$$
(2 \pi)^{-1}\left(1-x^{2}\right)^{-1 / 2} d x \leq d \nu_{\infty}(x) \leq(2 \pi)^{-1}\left(a^{2}-x^{2}\right)^{-1 / 2} d x .
$$


Our goal here is to prove the following, which we show implies Theorem 7.1 as a corollary.

THEOREM 7.2

Let $d \mu_{1}, d \mu_{2}$ be two measures on $\mathbb{R}$ of compact support. Suppose that for some interval $I=(\alpha, \beta)$,

(i)

$$
d \mu_{1} \leq d \mu_{2}
$$

(ii)

$$
d \mu_{1} \uparrow(\alpha, \beta)=d \mu_{2} \uparrow(\alpha, \beta)
$$

Let $n(j) \rightarrow \infty$, and suppose that $d \nu_{n(j)}^{(k)} \rightarrow d \nu_{\infty}^{(k)}$ for $k=1,2$, where $d v_{n}^{(k)}$ is the zero counting measure for $d \mu_{k}$. Then on $(\alpha, \beta)$,

$$
d v_{\infty}^{(2)} \uparrow(\alpha, \beta) \leq d v_{\infty}^{(1)} \uparrow(\alpha, \beta)
$$

Proof

By (1.12),

$$
\lambda_{n}\left(x, d \mu_{1}\right) \leq \lambda_{n}\left(x, d \mu_{2}\right),
$$

so by (1.14),

$$
\frac{1}{n+1} K_{n}^{(2)}(x, x) \leq \frac{1}{n+1} K_{n}^{(1)}(x, x)
$$

for all $x$. By (7.4) on $(\alpha, \beta)$,

$$
\frac{1}{n+1} K_{n}^{(2)}(x, x) d \mu_{2} \leq \frac{1}{n+1} K_{n}^{(1)}(x, x) d \mu_{1} .
$$

By Theorem 2.4, this implies (7.5).

Proof of Theorem 7.1

Let $d \mu_{2}=d \mu$, and let $d \mu_{1}=\chi_{(-a, a)}[d \mu]$ so that $d \mu_{1} \leq d \mu_{2}$ with equality on $[-a, a]$. By Theorem 5.1, $d \mu_{1}$ is regular for $[a, a]$, so $d v_{n}^{(2)} \rightarrow(2 \pi)^{-1}\left(a^{2}-\right.$ $\left.x^{2}\right)^{-1 / 2} d x$, the equilibrium measure for $[-a, a]$. Thus (7.5) implies the second inequality in (7.2).

On the other hand, let $d \mu_{1}=d \mu$, and let $d \mu_{2}=\left[\chi_{(-1,1)}-\chi_{(-a, a)}\right] d x+d \mu$. Then $d \mu_{1} \leq d \mu_{2}$ with equality on $(-a, a)$. Moreover, $d \mu_{2}$ is regular for $[-1,1]$, by Theorem 5.1 and the hypothesis $\sigma(d \mu) \subset[-1,1]$. Thus $d \nu_{n}^{(1)} \rightarrow(2 \pi)^{-1}(1-$ $\left.x^{2}\right)^{-1 / 2} d x$. Thus (7.5) implies the first inequality in (7.2). 


\section{Remarks}

(1) Theorem 7.1 only requires $\sigma_{\text {ess }}(d \mu) \subset[-1,1]$.

(2) The theorems in [35] are weaker than Theorem 7.1 in one respect and stronger in another. They are weaker in that because of their dependence on potential theory, they require that one of the comparison measures be regular. On the other hand, they are stronger in that our reliance on weak convergence limits us to open sets like $(\alpha, \beta)$, while they can handle more general sets.

(3) In [26], there is an example of a measure, $d \mu$, on $[-1,1]$, where $w(x)>0$ on $[-1,0]$ and the zero counting measures include among their limit points the equilibrium measures for $[-1,0]$ and for $[-1,1]$. This shows that in the $[a, b]$-form of Theorem 7.1, both inequalities in (7.2) can be saturated.

\section{Totik's bound for OPUC}

As preparation for applying our strategy to subsets of $\partial \mathbb{D}$, we need to prove an analog of Totik's bound (1.23) for closed sets on $\partial \mathbb{D}$. Given $a, b \in \partial \mathbb{D}$, we let $I=(a, b)$ be the interval of all points between $a$ and $b$, that is, going counterclockwise from $a$ to $b$, so $-1 \in\left(e^{i \theta}, e^{i(2 \pi-\theta)}\right)$ for $0<\theta<\pi$ but $-1 \notin\left(e^{i(2 \pi-\theta)}, e^{i \theta}\right)$. Given $E \subset \partial \mathbb{D}$ closed, we let $d \rho_{E}$ be its equilibrium measure. If $I \subset E \subset \partial \mathbb{D}$ is a nonempty open interval, then

$$
d \rho_{E} \uparrow I=\rho_{E}(\theta) d m(\theta)
$$

where $d m=\frac{d \theta}{2 \pi}$. The main theorem of this section is the following.

\section{THEOREM 8.1}

Let $I \subset E \subset \partial \mathbb{D}$, where $I=(a, b)$ is an interval and $E$ is closed. Let $d \mu$ be a measure with support in $E$ so that

$$
d \mu(\theta)=w(\theta) d m+d \mu_{\mathrm{s}}(\theta) .
$$

Then for $d m$-a.e. $\theta \in I$, we have

$$
\limsup n \lambda_{n-1}\left(e^{i \theta}\right) \leq \frac{w(\theta)}{\rho_{E}(\theta)} .
$$

Following Totik's strategy in [33] and [34] for OPRL, we do this in two steps.

\section{THEOREM 8.2}

(8.3) holds if $\operatorname{supp}(d \mu) \subset E^{\mathrm{int}}$ and $E$ is a finite union of intervals whose relative harmonic measures are rational. 


\section{THEOREM 8.3}

For any closed $E$ in $\partial \mathbb{D}$, we can find $E_{n}$ with the following.

(i) Each $E_{n}$ is a finite union of intervals whose relative harmonic measures are rational.

(ii)

$$
E \subset E_{n}^{\mathrm{int}}
$$

(iii)

$$
C\left(E_{n} \backslash E\right) \rightarrow 0
$$

Remark. If $E=I_{1} \cup \cdots \cup I_{\ell}$, the relative harmonic measures are $\rho_{E}\left(I_{j}\right)$, which sum to 1 .

Proof of Theorem 8.1, given Theorems 8.2 and 8.3

By general principles, since $I$ is an interval, $\rho_{E_{n}}(\theta)$ and $\rho_{E}(\theta)$ are real analytic with bounded derivatives. We have that (8.5) implies that $d \rho_{E_{n}} \stackrel{w}{\longrightarrow} d \rho_{E}$, and then the bounded derivative implies that $\rho_{E_{n}}(\theta) \rightarrow \rho_{E}(\theta)$ uniformly on compact subsets of $I$. By Theorem 8.2, the left-hand side of $(8.3) \leq w(\theta) / \rho_{E_{n}}(\theta)$ for each $n$. Since $\rho_{E_{n}}(\theta) \rightarrow \rho_{E}(\theta)$, we obtain (8.3).

Our proof of Theorems 8.2 and 8.3 diverges from the Totik strategy in two ways. Totik obtains Theorem 8.2 by using polynomial maps. Instead, following Simon [28], we use Floquet solutions.

Second, Totik shows if $E$ has $\ell$ gaps, one can find $E_{n}$ with rational relative harmonic measures also with $\ell$ gaps obeying (8.4) and (8.5). This is a result with rather different proofs by Bogatyrëv [3], Peherstorfer [19], and Totik [34]. I believe that any of these proofs extends to OPUC, but we settle for a weaker result: our $E_{n}$ 's contain up to $2 \ell$ intervals, the first $\ell$ each containing one of the $\ell$ intervals of $E$ and an additional $\ell$ or fewer exponentially small intervals. This allows us to get away with following only the easier part of Peherstorfer's strategy.

\section{Proof of Theorem 8.2}

By [24, Theorem 11.4.5], $E$ is the essential spectrum of an isospectral torus of Verblunsky coefficients periodic up to a phase; that is, for suitable $p$ (chosen so that $p \rho_{E}\left(I_{j}\right)$ is an integer for each $\left.j\right)$,

$$
\alpha_{n+1}=\lambda \alpha_{n}
$$

Let $\mu_{E}$ be the measure associated to a point on the isospectral torus. 
By Floquet theory (see [24, Sec. 11.2]), for $z \in E^{\text {int }}, \varphi_{n}$ is a sum of two functions each periodic up to a phase, and by [24, Sec. 11.12], on compact subsets, $K$, of $E^{\text {int }}$,

$$
\sup _{z \in K, n}\left|\varphi_{n}(z)\right|<\infty
$$

It follows from the CD formula (see [23, Sec. 2.2]) that

$$
\sup _{z, w \in K}\left|K_{n}(z, w)\right| \leq C|z-w|^{-1} \text {. }
$$

The almost periodicity of $\varphi$ implies that uniformly on $K,(1 /(n+1)) K_{n}(z, z)$ has a finite nonzero limit, and then, by Theorem 2.7, the limit must be $\rho_{E}(\theta) / w_{E}(\theta)$; that is, uniformly on $K$,

$$
\lim _{n \rightarrow \infty} \frac{1}{n} K_{n}\left(e^{i \theta}, e^{i \theta}\right)=\frac{\rho_{E}(\theta)}{w_{E}(\theta)}
$$

where $w_{E}(\theta)$ is the weight for $d \rho_{E}(\theta)$. Moreover, as proved in Simon [28], for any $A>0$, uniformly on $e^{i \theta} \in K$ and $n|\varphi-\theta|<A$,

$$
Q_{n}\left(e^{i \varphi}\right) \equiv \frac{K_{n}\left(e^{i \theta}, e^{i \varphi}\right)}{K_{n}\left(e^{i \theta}, e^{i \theta}\right)}=\frac{\sin \left(n \rho_{E}(\theta)(\theta-\varphi)\right)}{n(\theta-\varphi) \rho_{E}(\theta)}(1+O(1))
$$

Now, use $Q_{n}\left(e^{i \varphi}\right)$ as a trial function in (1.12). By (8.7) and (8.8), by taking $A$ large, the contribution of $n|\varphi-\theta|>A$ can be made arbitrarily small. Maximal function arguments and (8.9) show that the contribution of the region $n|\varphi-\theta|<A$ to $n \lambda_{n-1}$ is close to $w(\theta) / \rho_{E}(\theta)$. This proves $(8.3)$ for $d \mu$.

Given $E \subset \partial \mathbb{D}$ compact, define

$$
\tilde{E}_{n}=\left\{e^{i \theta} \in \mathbb{D} \mid \operatorname{dist}\left(e^{i \theta}, E\right) \leq \frac{1}{n}\right\}
$$

It is easy to see that $C\left(\tilde{E}_{n} \backslash E\right) \rightarrow 0$ and that $\tilde{E}_{n}$ is a union of $\ell(n)<\infty$ closed intervals. It thus suffices to prove Theorem 8.3 when $E$ is already a union of finitely many $\ell$ disjoint closed intervals, and it is that which we are heading toward. (Parenthetically, we note that we could dispense with this and instead prove the analog of Theorem 8.2 for a finite union of intervals using Jost solutions for the isospectral torus associated to such finite gap sets, as in Simon [28].)

Define $\mathscr{P}_{n}$ to be the set of monic polynomials, all of whose zeros lie in $\partial \mathbb{D}$. Since

$$
e^{i \theta / 2}+e^{i \varphi} e^{-i \theta / 2}=e^{i \varphi / 2}\left[e^{i(\theta-\varphi) / 2}+e^{-i(\theta-\varphi) / 2}\right],
$$


if $P \in \mathscr{P}_{n}$, there is a phase factor $e^{i \eta}$, so that

$$
z^{-n / 2} e^{i \eta} P(z) \text { is real on } \partial \mathbb{D} \text {. }
$$

Define the restricted Chebyshev polynomials, $\tilde{T}_{n}$, associated to $E \subset \partial \mathbb{D}$ by requiring that $\tilde{T}_{n}$ minimize

$$
\left\|P_{n}\right\|_{E}=\sup _{z \in E}\left|P_{n}(z)\right|
$$

over all $P_{n} \in \mathscr{P}_{n}$. We show that for $n$ large, $2 \tilde{T}_{2 n} /\left\|\tilde{T}_{2 n}\right\|_{E}$ are the rotated discriminants associated to sets $E_{n}$ that approximate an $E$ that is a finite union of intervals. An important input is the following.

LEMMA 8.4

Let $I=\left(z_{0}, z_{1}\right)$ be an interval in $\partial \mathbb{D}$. For any small $\varphi$, let $I_{\varphi}=\left(z_{0} e^{i \varphi}, z_{1} e^{-i \varphi}\right)$, so for $\varphi>0, I_{\varphi}$ is smaller than I. For $\varphi<0$,

$$
\left|\left(z-z_{0} e^{i \varphi}\right)\left(z-z_{1} e^{-i \varphi}\right)\right|
$$

decreases on $\partial \mathbb{D} \backslash I$ and increases on I as $\varphi$ decreases in $(-\varepsilon, 0)$.

\section{Proof}

Take $z_{1}=\bar{z}_{0}$, and then use some elementary calculus.

\section{THEOREM 8.5}

Let $E$ be a finite union of disjoint closed intervals on $\partial \mathbb{D}, E=I_{1} \cup \cdots \cup I_{\ell}$, and let $\partial \mathbb{D} \backslash E=G_{1} \cup \cdots \cup G_{\ell}$ have $\ell$ gaps. Then we have the following.

(i) Each $\tilde{T}_{n}$ has at most one zero in each $G_{\ell}$.

(ii) If $z_{j}^{(n)}$ is the zero of $\tilde{T}_{n}$ in $G_{j}$, then on any compact $K \subset G_{j}$, we have

$$
\lim _{n \rightarrow \infty} \inf _{z \in K}\left(\left|\frac{\tilde{T}_{n}(z)}{\left(z-z_{j}^{(n)}\right)\left\|T_{n}\right\|_{E}}\right|\right)^{1 / n}>1 .
$$

(iii) At any local maximum, $\tilde{z}$, of $\left|\tilde{T}_{n}(z)\right|$ in some $I_{j}$, we have

$$
\left|\tilde{T}_{n}(\tilde{z})\right|=\left\|\tilde{T}_{n}\right\|_{E}
$$

\section{Proof}

(i) If there are two zeros in some $G_{j}$, we can symmetrically move the zeros apart. Doing that increases $\tilde{T}_{n}$ on $G_{j}$, which is disjoint from $E$, but it decreases $\left\|\tilde{T}_{n}\right\|_{E}$, contradicting the minimizing definition. 
(ii) The zero counting measure for $\tilde{T}_{n}$ converges to the equilibrium measure on $E$. For standard $T_{n}$ 's and $E \subset \mathbb{R}$, this result is proved in [1], [22], [26]. A small change implies this result for $\tilde{T}_{n}$. This, in turn, says that locally uniformly on $G_{j}$,

$$
\left|\frac{\tilde{T}_{n}(z)}{\left(z-z_{j}^{(n)}\right)\left\|\tilde{T}_{n}\right\|_{E}}\right|^{1 / n} \rightarrow \exp \left(-\Phi_{\rho_{E}}(z)\right),
$$

which implies (8.13).

(iii) Since $z^{-n / 2} \tilde{T}_{n}(z)$ is real up to a phase, the local maxima of $\left|\tilde{T}_{n}(z)\right|$ on $\partial \mathbb{D}$ alternate with the zeros of $\tilde{T}_{n}(z)$. If a local maximum is smaller than $\left\|\tilde{T}_{n}\right\|_{E}$, we move the nearest zeros, say, $z_{0}, z_{1}$, apart. That decreases $\left\|\tilde{T}_{n}\right\|_{E \backslash\left(z_{0}, z_{1}\right)}$ and increases $\left\|\tilde{T}_{n}\right\|_{\left(z_{0}, z_{1}\right)}$. Since the latter is assumed smaller than $\left\|\tilde{T}_{n}\right\|_{E}$, it decreases $\left\|\tilde{T}_{n}\right\|_{E}$ overall, violating the minimizing definition. Thus, $\left|\tilde{T}_{n}(z)\right| \geq\left\|\tilde{T}_{n}\right\|_{E}$. But since $\tilde{z} \in E,\left|\tilde{T}_{n}(z)\right| \leq\left\|\tilde{T}_{n}\right\|_{E}$.

Now, define

$$
\Delta_{n}(z)=\frac{2 e^{i \varphi_{n}} \tilde{T}_{2 n}(z)}{\left\|\tilde{T}_{2 n}\right\|_{E}},
$$

where $\varphi_{n}$ is chosen to make $\Delta_{n}$ real on $\partial \mathbb{D}$. By (8.14), maxima in $E$ occur with $\Delta_{n}(z)= \pm 2$, and by (8.13), maxima in $\partial \mathbb{D} \backslash E$ occur at points where $\left|\Delta_{n}(z)\right|>2$. Thus, up to a phase, $\Delta_{n}(z)$ looks like a discriminant. So, by [24, Theorem 11.4.5], $E_{n} \equiv \Delta_{n}^{-1}([-2,2])$ is the essential spectrum of a CMV matrix whose Verblunsky coefficients obey $\alpha_{m+p}=\lambda \alpha_{m}$ for $|\lambda|=1$.

\section{Proof of Theorem 8.3}

$E_{n}$ has at most $2 \ell$ components, $\ell$ containing $I_{1}, \ldots, I_{\ell}$ (call them $I_{1}^{(n)}, \ldots, I_{\ell}^{(n)}$ ), and $\ell$ possible components, $J_{1}^{(n)}, \ldots, J_{\ell}^{(n)}$, one in each gap. Since capacities are bounded by one-quarter times the Lebesgue measure, it suffices to show that

$$
\sum_{j=1}^{\ell}\left|I_{j}^{(n)} \backslash I_{j}\right|+\left|J_{j}^{(n)}\right| \rightarrow 0
$$

to prove (8.5) and complete the proof. Since, on $\partial \mathbb{D}$,

$$
-\Phi_{\rho_{E}}(x) \geq c \operatorname{dist}(x, E)^{1 / 2}
$$

by (8.15), we have $\left|I_{j}^{(n)} \backslash I_{j}\right| \rightarrow 0$ and $\left|J_{j}^{(n)}\right| \rightarrow 0$. 


\section{Theorems for subsets of $\partial \mathbb{D}$}

Given Theorem 8.1 and our strategies in Sections 3-6, we immediately have the following.

\section{THEOREM 9.1}

Let $E \subset \partial \mathbb{D}$ with $\partial E=E \backslash E^{\mathrm{int}}\left(E^{\mathrm{int}}\right.$ means interior in $\left.\partial \mathbb{D}\right)$ having capacity zero. Let $d \mu$ be a measure on $\partial \mathbb{D}$ with $\sigma_{\mathrm{ess}}(d \mu)=E$ and

$$
d \mu=f(x) d \rho_{E}+d \mu_{s}
$$

where $d \mu_{s}$ is $d \rho_{E}$-singular. Suppose that $f(x)>0$ for $d \rho_{E}$-a.e. $x$. Then $d \mu$ is regular.

THEOREM 9.2

Let $I \subset E \subset \partial \mathbb{D}$ with I a nonempty closed interval and $E$ closed. Let $d \mu$ be a measure on $\partial \mathbb{D}$ so that $\sigma_{\mathrm{ess}}(d \mu)=E$ and $\mu$ is regular for $E$. Suppose that

$$
d \mu=w(\theta) \frac{d \theta}{2 \pi}+d \mu_{s},
$$

and suppose that $w(\theta)>0$ for a.e. $e^{i \theta} \in I$. Then

(i)

$$
\frac{1}{n+1} K_{n}\left(e^{i \theta}, e^{i \theta}\right) d \mu_{\mathrm{s}}(\theta) \stackrel{w}{\longrightarrow} 0
$$

(ii)

$$
\int_{I}\left|\rho_{E}(\theta)-\frac{1}{n+1} w(\theta) K_{n}\left(e^{i \theta}, e^{i \theta}\right)\right| \frac{d \theta}{2 \pi} \rightarrow 0,
$$

where $d \rho(\theta)=\rho_{E}(\theta) \frac{d \theta}{2 \pi}$ on $I$.

Acknowledgments. It is a pleasure to thank Jonathan Breuer, Yoram Last, and especially Vilmos Totik for useful conversations. I also thank Ehud de Shalit and Yoram Last for the hospitality of the Einstein Institute of Mathematics of the Hebrew University during part of the preparation of this article.

\section{References}

[1] V. V. ANDRIEVSKII and H.-P. BLATT, Discrepancy of Signed Measures and Polynomial Approximation, Springer Monogr. Math., Springer, New York, 2002. MR 1871219 
[2] J. AVRON and B. SIMON, Almost periodic Schrödinger operators, II: The integrated density of states, Duke Math. J. 50 (1983), 369-391. MR 0700145

[3] A. B. BOGATYRËV, On the efficient computation of Chebyshev polynomials for several intervals (in Russian), Mat. Sb. 190 no. 11 (1999), 15 - 50; English translation in Sb. Math. 190 (1999), 1571 - 1605. MR 1735137

[4] M. J. CANTERO, L. MORAL, and L. VELÁZQUEZ, Measures and para-orthogonal polynomials on the unit circle, East J. Approx. 8 (2002), 447-464. MR 1952510

[5] - Five-diagonal matrices and zeros of orthogonal polynomials on the unit circle, Linear Algebra Appl. 362 (2003), 29-56. MR 1955452

[6] - Measures on the unit circle and unitary truncations of unitary operators, J. Approx. Theory 139 (2006), 430 - 468. MR 2220048

[7] E. B. CHRISTOFFEL, Über die Gaussische Quadratur und eine Verallgemeinerung derselben, J. Reine Angew. Math. 55 (1858), 61 - 82.

[8] G. DARBOUX, Mémoire sur l'approximation des fonctions de très-grands nombres, et sur une classe étendue de développements en série, J. Math. Pures Appl. 4 (1878), $5-56 ; 377-416$.

[9] E. B. DAVIES and B. SIMON, unpublished manuscript.

[10] P. ERDÖS and P. TURÁN, On interpolation, III: Interpolatory theory of polynomials, Ann. of Math. (2) 41 (1940), 510 - 553. MR 0001999

[11] G. FREUD, Orthogonal Polynomials, Pergamon Press, Oxford Univ. Press, New York, 1971.

[12] Y. L. GERONIMUS, Orthogonal Polynomials: Estimates, Asymptotic Formulas, and Series of Polynomials Orthogonal on the Unit Circle and on an Interval, Consultants Bureau, New York, 1961. MR 0133643

[13] L. GOLINSKII, Quadrature formula and zeros of para-orthogonal polynomials on the unit circle, Acta Math. Hungar. 96 (2002), 169 - 186. MR 0919160

[14] L. GOLINSKII and S. KHRUSHCHEV, Cesàro asymptotics for orthogonal polynomials on the unit circle and classes of measures, J. Approx. Theory 115 (2002), 187-237. MR 1901215

[15] W. B. JONES, O. NJÅSTAD, and W. J. THRON, Moment theory, orthogonal polynomials, quadrature, and continued fractions associated with the unit circle, Bull. London Math. Soc. 21 (1989), 113 - 152. MR 1976057

[16] A. MÁTÉ and P. G. NEVAI, Bernstein's inequality in $L^{p}$ for $0<p<1$ and $(C, 1)$ bounds for orthogonal polynomials, Ann. of Math. (2) 111 (1980), 145 - 154. MR 0558399

[17] A. MÁTÉ, P. G. NEVAI, and V. TOTIK, Strong and weak convergence of orthogonal polynomials, Amer. J. Math. 109 (1987), 239-281. MR 0882423

[18] - Szegö's extremum problem on the unit circle, Ann. of Math. (2) 134 (1991), 433-453. MR 1127481

[19] F. PEHERSTORFER, Deformation of minimal polynomials and approximation of several intervals by an inverse polynomial mapping, J. Approx. Theory 111 (2001), 180 - 195. MR 1849545

[20] E. A. RAHMANOV [RAKHMANOV], The asymptotic behavior of the ratio of orthogonal polynomials (in Russian), Mat. Sb. (N.S.) 103(145), no. 2, 237-252; English translation in Math. USSR Sb. 32 (1977), 199-213. MR 0445212 
[21] - The asymptotic behavior of the ratio of orthogonal polynomials, II (in Russian), Mat. Sb. (N.S.) 118(160), no. 1 (1982), $104-117$; English translation in Math. USSR Sb. 46 (1983), 105 - 117. MR 0654647

[22] E. B. SAFF and V. TOTIK, Logarithmic Potentials with External Fields, Grundlehren Math. Wiss. 316, Springer, Berlin, 1997. MR 1485778

[23] B. SIMON, Orthogonal Polynomials on the Unit Circle, Part 1: Classical Theory, Amer. Math. Soc. Colloq. Publ. 54, Part 1, Amer. Math. Soc., Providence, 2005. MR 2105088

[24] - Orthogonal Polynomials on the Unit Circle, Part 2: Spectral Theory, Amer. Math. Soc. Colloq. Publ. 54, Part 2, Amer. Math. Soc., Providence, 2005. MR 2105089

[25] - CMV matrices: Five years after, J. Comput. Appl. Math. 208 (2007), 120 - 154. MR 2347741

[26] Equilibrium measures and capacities in spectral theory, Inverse Probl. Imaging 1 (2007), 713 - 772. MR 2350223

[27] Rank one perturbations and the zeros of paraorthogonal polynomials on the unit circle, J. Math. Anal. Appl. 329 (2007), 376-382. MR 2306808

[28] - Two extensions of Lubinsky's universality theorem, to appear in J. Anal. Math.

[29] - Szegö's Theorem and Its Descendants: Spectral Theory for $L^{2}$ Perturbations of Orthogonal Polynomials, forthcoming from Princeton Univ. Press.

[30] H. STAHL and V. TOTIK, General Orthogonal Polynomials, Encyclopedia Math. Appl. 43, Cambridge Univ. Press, Cambridge, 1992. MR 1163828

[31] G. SZEGÖ, Beiträge zur Theorie der Toeplitzschen Formen, I, Math. Z. 6 (1920), 167-202; II, 9 (1921), 167-190. MR 1544404; MR 1544462

[32] - Orthogonal Polynomials, 3rd ed., Amer. Math. Soc. Colloq. Publ. 23, Amer. Math. Soc., Providence, 1967. MR 0310533

[33] V. TOTIK, Asymptotics for Christoffel functions for general measures on the real line, J. Anal. Math. 81 (2000), 283-303. MR 1785285

[34] - Polynomial inverse images and polynomial inequalities, Acta Math. 187 (2001), 139-160. MR 1864632

[35] V. TOTIK and J. L. ULLMAN, Local asymptotic distribution of zeros of orthogonal polynomials, Trans. Amer. Math. Soc. 341 (1994), 881-894. MR 1150019

[36] W. VAN ASSCHE, Invariant zero behaviour for orthogonal polynomials on compact sets of the real line, Bull. Soc. Math. Belg. Sér. B 38 (1986), 1 - 13. MR 0871299

[37] H. WIDOM, Polynomials associated with measures in the complex plane, J. Math. Mech. 16 (1967), 997 - 1013. MR 0209448

[38] M. L. WONG, First and second kind paraorthogonal polynomials and their zeros, J. Approx. Theory 146 (2007), 282 - 293. MR 2328186

Department of Mathematics, California Institute of Technology, Pasadena, California 91125, USA; bsimon@caltech.edu 